\title{
Research on the Development Strategy of National Fitness Sports in Northwest China
}

\author{
Peng Xiao \\ College of Physical Education, Northwest Normal University, Lanzhou, Gansu 730070, China \\ xiaopeng1949@126.com
}

Keywords: Northwest China; National fitness; Development strategy; Ideas

\begin{abstract}
This study uses literature research, questionnaire survey, expert interviews, history and logic to analyze the current situation of national fitness and sports in the northwest region of China and the internal and external environment. It also explores the development opportunity, and puts forward the idea of the development of national fitness sports in the northwest region.
\end{abstract}

\section{Introduction}

There are obvious "gradient differences" in the level of economic and social development in the western, central and eastern regions of China. This "gradient difference" is the realistic basis for the development of regional sports. Due to the differences in economic, cultural and social developments, unique regional cultural phenomena and behavioral habits have been formed. National fitness sports will also show differences in the development of different environments. Accordingly, in accordance with the requirements of the scientific development concept, combined with the goals and tasks of building a well-off society in an all-round way and building a diversified national fitness service system, we will measure the actual state of the national fitness sports in the northwest region, identify gaps, and identify development problems, constraints and priorities. Exploring the laws and trends of the development of mass sports, which has important theoretical significance and realistic strategic significance for accelerating the development of sports in the northwest and the in-depth development of national fitness and sports, and the realization of the goal of building a well-off society in an all-round way.

\section{Research ideas and framework}

This study intends to use the following four aspects as the theoretical fulcrum of the research framework design.

(1) Analysis of the economic and social environment (external environment) of the national fitness and sports center in the northwest region. Since the reform and opening up, China's mass sports have made extraordinary achievements. However, we cannot deny the fact that there are obvious "gradient differences" in the social culture and economic development level in the eastern, central and western regions of China. This "gradient difference" determines the development of national fitness sports in different regions. Adopt different strategic options. 
(2) Current situation and practical problems of national fitness sports in Northwest China. Due to the differences in economic, cultural and social environment, the characteristics of China's regional cultural atmosphere and behavioral habits have been formed. The national fitness sports will show differences in the development of various environments, and gradually form new sports forms with regional characteristics. Its localization and development under different cultural backgrounds will become the possibility and necessity of development. At the same time, we need to measure the actual state of the national fitness sports in the northwest region under the current economic and social conditions for the specific requirements of the national fitness sports, so as to find the gap and determine the problem.

(3) Opportunities for the development of national fitness sports in the Northwest. The report of the 16th National Congress of the Communist Party of China pointed out that the first 20 years of the 21st century is an important strategic opportunity period for China to grasp and can make a difference. This is an extremely important judgment. We can grasp from many aspects the basis of the theoretical exploration of the National Fitness Sports Department in Northwest China during the important strategic opportunity period.

(4) In the next 5-10 years, the strategic goals of the development of national fitness sports in the northwest region (including overall development goals and specific targets) and major strategic countermeasures.

\section{Analysis of the economic and social environment in the Northwest}

At present, the theoretical community has reached a consensus on the development of sports subject to the development level of economy, society and sports itself. Through the analysis of relevant research and research results, expert consultation and field research, the research team believes that the economic and social environment affecting the development of national fitness sports in the northwest region is mainly as follows: (1) low level of economic development and reliance on various consumer markets. The economic foundation is weak; (2) the level of urbanization is low, and the population concentration is not high; (3) the development of social undertakings lags behind, and the cultural level of rural residents is generally not high; (4) the proportion of minority population is high, and the spatial distribution of population is extremely low. Equilibrium; (5) Poverty issues are more prominent, and poverty eradication has a long way to go ${ }^{[1-3]}$.

\section{Current Situation and Realistic Problems of National Fitness Sports in Northwest China}

Nationality is one of the basic characteristics of the national fitness system (Dong Xinguang, 2005), which is determined by the goal of "building a well-off society for more than one billion people" and the nature of "all the people" in the "national fitness system". Based on the actual situation in the northwest region, the research team designed 6 kinds of questionnaires for the service targets and benefit recipients of the national fitness system, distributed more than 6,000 questionnaires, and visited more than 100 people (households) of the citizens and the Chinese. The urban community sports in the northwest, the national fitness sports in rural areas, the rural township sports in the Hui community, the school sports in the poverty-stricken minority areas, and the current situation of urban residents' sports consumption were investigated and analyzed.

\subsection{The development of rural fitness in the northwestern region}

The rural fitness infrastructure, sports population, farmers' physical fitness awareness, sports consumption level, mass sports organization and management are still at a relatively low level, 
highlighting the development in the process of building a well-off society in an all-round way. The arduousness and importance of national fitness and sports in the region. At the same time, in the great historical process of China's commitment to western development, building a well-off society in an all-round way, and building a socialist harmonious society, rural areas in the northwestern region with diverse sports culture and rich sports tourism resources have good opportunities in developing national fitness and sports ${ }^{[4]}$.

\subsection{Status of sports development in rural towns and villages in the northwest Hui nationality area}

Mainly manifested as: lack of physical activity venue facilities; township government departments pay insufficient attention; lack of sports activities funds; township residents are not enthusiastic about carrying out sports activities; lack of guidance for sports activities; lack of unified organizational management, etc. is currently the Northwest Hui The main difficulties faced by rural townships in rural areas ${ }^{[5] .}$

\subsection{Status of urban community sports in the Northwest}

The development of community sports organizations is relatively mature; the number of community sports instructors is relatively small; the leisure time of urban residents is mainly watching TV, and the stadium is not very motivated; the exercises are based on walking, chess, Tai Chi, ballroom dancing, and goalball. The main event time is morning and evening; the city square is the main place for residents' sports activities, and most of the stadiums are not open to the public ${ }^{[6]}$.

\subsection{Status quo of school sports development in poor areas of poverty-stricken ethnic groups in Northwest China}

Most schools have established physical education classes; the number of physical education teachers is relatively insufficient; there are widespread problems of serious shortage of venues and equipment; students are highly motivated to participate in sports activities; primary school students are mainly engaged in sports games with small exercise intensity, and middle school students The baskets are mainly based on projects, and the development of local textbooks has not received enough attention ${ }^{[7]}$.

\subsection{Current Status of Sports Consumption of Urban Residents in Northwest China}

Sports consumption has become a part of urban residents' living consumption; urban residents are basically in favor of sports consumption; physical fitness ranks first in residents' sports consumption; current sports consumption level is generally not high, and physical consumption consumption is in physical consumption Mainly; the factors affecting residents' sports consumption are: income level, ember time, venue facilities, sports consumption awareness and so on ${ }^{[8] .}$

\section{Strategic Plan for the Development of National Fitness Sports in Northwest China}

This is an important period of strategic opportunity that must be firmly grasped and can make a difference. For the National Fitness and Sports Division in Northwest China, we can make a significant basis for the important strategic opportunities. We can grasp from the following aspects: the rapid development of the economy and society provides a solid material and site facilities guarantee; The mass sports activities have improved the awareness of sports participation of the 
people in the vast areas; the reform of the sports system has provided more effective institutional guarantees and policy support for the development of national fitness sports in the northwestern region; the rapid development of various rural undertakings.

\subsection{Strategic Objectives of the Development of National Fitness Sports in Northwest China in the Future 5-10 Years}

Overall development goals. In the next 5-10 years, the northwestern region of China should improve the physical quality, health level and quality of life of all ethnic groups and all walks of life, and promote the all-round development of people as a starting point and a foothold to build a diverse public sport with regional characteristics. The service system is the main line, further consolidating the social foundation of sports development, taking the innovation of sports system as the driving force, strengthening the public sports service function, and comprehensively promoting the development of the national fitness industry as the overall development goal.

Specific target. After 5-10 years of hard work, the awareness of physical fitness of urban and rural residents in northwest China has been further enhanced. The number of people participating in physical exercise has increased significantly, and the physical quality has improved significantly. A public health service system covering the urban and rural areas has been formed. The specific performance is: the number of people participating in physical exercise is further increased, the physical quality of urban and rural residents is further improved, the physical fitness facilities are greatly developed, the content of national fitness activities is more abundant, the network of national fitness organizations is more complete, the fitness guidance of the whole people and the volunteer service team are further developed. The scientific fitness guidance service has been continuously improved, and the national fitness service industry has grown stronger.

\subsection{Main Strategic Tasks for the Development of National Fitness Sports in Northwest China in the Future 5-10 Years}

The goal of building a well-off society in an all-round way will bring unprecedented development opportunities and challenges to the national fitness sports in northwestern China. Then, in such a good situation, the northwest region should develop the national fitness sports in order to protect the broad masses of the people. Sports services." We believe that the national fitness and sports must be implemented in the end. Only in accordance with the various requirements of the new era for sports development, bravely face challenges and constraints, as well as the actual situation in the northwest region, and conscientiously resolve the long-term accumulation and existence of outstanding contradictions. And problems, break through bottlenecks and institutional barriers to development, focus on the scope and target system of the implementation of the National Fitness Program, accurately analyze the characteristics of the participating groups, and effectively focus on schools, cities, rural areas, and ethnic areas with the largest minority population in the country. With the construction and activities of the national fitness and sports, we will be able to create a new situation in the modernization of sports in the northwest region.

\section{Conclusion}

The development of mass sports is one of the important indicators to measure the level of sports and social and cultural development of a country or a nation. This study focuses on the reality of China's economic and social development in the northwest region, analyzes and summarizes the development status and development opportunities of the national fitness sports in the northwest region, and discusses the strategic goals and tasks of the national fitness sports development in the 
region. It is expected to benefit the urban and rural development and regional development of sports.

\section{Acknowledgement}

This article is the National Social Science Fund Western Project "Study on the Development Strategy of National Fitness Sports in Northwest China in the Process of Building a Well-off Society in an All-round Way" (Project No. 05XTY004); Gansu Sports Social Science Research Youth Project (Project No.: GST201338); Northwest Normal University The final report of the Young Teacher Research Fund Project (Project No.: NMNU-SKQN-09-34).

\section{References}

[1] Dong Suocheng. Comparative Advantages in Northwest China and Regional Economic Development[M]. Lanzhou: Gansu People's Publishing House, Vol. 5 (2001) No.18, p. 107-108.

[2] Zhang Weiqing. Commanding the Population Development in Northwest China with Scientific Development View. Population and Family Planning, Vol. 4 (2005) No.10, p. 123-125.

[3] Dong Xinguang. Theoretical framework of the national fitness system. Sports Culture Guide, Vol. 5 (2005) No.5, p. 51-52.

[4] Li Runzhong. Research on the development countermeasures of national fitness sports in rural areas in Northwest China. Lanzhou: Northwest Normal University, Vol. 2 (2007) No.15, p. 124-127.

[5] Ma Xiaorong. Research on rural sports in rural areas in the northwest Hui people's area. Lanzhou: Northwest Normal University, Vol. 5 (2015) No.18, p. 12-15.

[6] Feng Tao. Research on the status quo and countermeasures of community sports in northwest China. Lanzhou: Northwest Normal University, Vol. 1 (2007) No.21, p.31-35.

[7] Zhong Quanhong. Research on School Sports in the Poor Minority Areas in Northwest China. Lanzhou: Northwest Normal University, Vol. 3 (2007) No.12, p.24-27.

[8] Xiong Bo. Research on the current situation of urban residents' sports consumption in Gansu Province. Lanzhou: Northwest Normal University, Vol. 23 (2007) No.12, p. 12-15. 